\title{
REPRESENTACIONES SOCIALES QUE TIENEN LOS DOCENTES DEL PROGRAMA DE EDUCACIÓN FÍSICA DE LA UNIVERSIDAD PEDAGÓGICA NACIONAL SOBRE LAS COMPETENCIAS PROPIAS DE SU SABER DISCIPLINAR SOCIAL REPRESENTATIONS OF THE PHYSICAL EDUCATION TEACHERS' AT UNIVERSIDAD PEDAGÓGICA NACIONAL REGARDING THEIR OWN KNOWLEDGE COMPETENCES
}

\author{
Luis Carlos Pérez Ferro ${ }^{1}$ \\ Martha Arenas Molina ${ }^{2}$ \\ Sonia López Domínguez ${ }^{3}$
}

Resumen

La investigación que se presenta describe las representaciones sociales que tienen los docentes del programa de Educación Física de la Universidad Pedagógica Nacional sobre las competencias propias de su saber disciplinar. Los presupuestos referenciales gravitan sobre la propuesta teórica de Moscovici y sus desarrollos ulteriores. Se utiliza el enfoque plurimetodológico, que refiere a la utilización de métodos interrogativos y asociativos basados en la expresión verbal. Los sujetos participantes del estudio son maestros de la Facultad de Educación Física de la Universidad Pedagógica Nacional. Los resultados mostraron que el núcleo central del corpus de la representación de la competencia se asocia a términos como: pensamiento complejo, capacidad, desarrollo, formación y aprehensión. Los docentes de Educación Física de la UPN concentran gran parte de su trabajo en el desarrollo de competencias básicas, circunscritas, fundamentalmente, a procesos cognitivos.

Palabras clave: Representaciones sociales, docentes, educación física, competencias profesionales, saber disciplinar.

Abstract

This research describes the social representations of PE teachers at Universidad Pedagógica Nacional regarding their own competencies applied to their discipline. The references are around both theoretical proposal and further developments by Moscovici. It uses a multi-methodological approach, which refers to survey and associative methods based on verbal expression. PE teachers from UPN are the descriptive analysis subjects. Results showed that core of the representation corpus are associated to terms, such as complex thinking, ability, development, training and grasp. Basically the teachers are focused on competencies development aimed to cognitive process.

Keywords: Social representations, teachers, physical education, professional competencies, discipline knowledge.

Fecha de recepción: 20 de junio de 2012

Fecha de aprobación: 24 de septiembre de 2012

1 Psicólogo, Universidad Santo Tomás, Bogotá. Magíster en Educación, Pontificia Universidad Javeriana, Bogotá. Humanista e investigador. Profesor asistente de la Facultad de Educación Física, Universidad Pedagógica Nacional. Grupo de estudios en Educación y Experiencia Corporal UPN. Correo electrónico: lcperez@pedagogica.edu.co

2 Licenciada en Lingüística y Literatura, Universidad Distrital Francisco José de Caldas. Especialista en Pedagogía y Docencia Universitaria, Universidad La Gran Colombia. Magistra en Educación, Pontificia Universidad Javeriana, Bogotá. Profesor asistente de la Facultad de Educación Física. Grupo de estudios en Educación y Experiencia Corporal UPN. Correo electrónico: marenas@pedagogica.edu.co 3 Profesora de planta, Facultad de Educación Física, Universidad Pedagógica Nacional. Magíster en Educación y Desarrollo Social, CINDE - UPN. Grupo de estudios en Educación y Experiencia Corporal UPN. Correo electrónico: solopez@pedagogica.edu.co 


\section{Introducción}

Se presentan en este artículo los hechos fundamentales del proceso investigativo, cuyo objeto central fue la descripción de las representaciones sociales que tienen los docentes del programa de Educación Física de la Universidad Pedagógica Nacional, sobre las competencias propias de su saber disciplinar.

El escrito está dividido en cuatro partes. La primera, llamada introducción, informa sobre la teoría de las representaciones sociales y subraya en ella aspectos característicos de su trabajo práctico; es decir, hace referencias a la forma mediante la cual se pueden abordar y describir. El segundo apartado es el método. Allí se presenta el diseño general de la investigación y se denota el carácter plurimetodológico del estudio. Luego están los resultados y por último las referencias bibliográficas.

\section{Representación social}

Las representaciones sociales son una propuesta teórica de origen francés, desarrollada en la segunda mitad del siglo XX por el rumano Serge Moscovici, quien dentro de su trabajo titulado El psicoanálisis, su imagen y su público, expone cómo la sociedad francesa de aquel entonces representa esta postura psicológica. Se conocen también desarrollos, incluso en el mismo Moscovici, de aspectos metodológicos cuya función es hacer de la teoría una práctica investigativa. Son, pues, teoría y método. En cuanto a lo primero, hay disponible una importante literatura; en cuanto a lo segundo, un gran camino por recorrer. En los distintos trabajos de investigación aplicada hay diversidad en las maneras de estudiar empíricamente las representaciones.

\section{Presupuestos centrales de la teoría}

1. Las representaciones sociales son parte del mundo de la interacción. La teoría se refiere a la vinculación que tenemos en el mundo social en tanto compartimos modos de pensar y actuar. Es, entonces, una propuesta de pensamiento social, una forma de explicar los lazos que nos unen tácitamente y que, en gran medida, matizan nuestras acciones cotidianas. En este sentido, no es posible referirse a una representación en solitario: será menester el otro. Es con él y mediante ello como se construyen, trasmutan y establecen los dominios del tejido social.

2. Las representaciones sociales son cognición social. Sobre el particular escribe Moscovici (1979): "La representación social es una modalidad particular de co- nocimiento, cuya función es la elaboración de los comportamientos y la comunicación entre los individuos".

Si admitimos que pensar es una facultad típicamente humana, entonces la representación social es una forma del pensar. Este proceso, ampliamente estudiado por la psicología, filosofía e incluso neurología, concibe, en términos globales, la comprensión acerca de cómo recibimos, procesamos y actuamos frente a la información y conocimiento derivado de la acción social y el legado de la herencia.

3. Las representaciones son conciencia social. Habitualmente el término conciencia se contrapone a no tenerla: inconciencia. En la medida en que identificamos, dentro del amplio espacio de contenidos sociales, una categoría o un concepto, lo actuamos y compartimos.

Es posible hacer consciente, reflectar, discernir y evaluar la realidad socialmente. Esta es una de las funciones de la representación: hacer inteligible el mundo. En tanto compartimos el ambiente social, es posible su conciencia. Sin embargo, no todo lo que se ha objetivado -se ha hecho consciente- es compartido. Baste mencionar cómo el infante, al otorgarle sentido a su realidad, oculta, saca del plano representacional lo que es poco significativo. La conciencia social, tan importante en los procesos de desarrollo, ocupa un lugar privilegiado en el análisis sociológico. Es ella la que permite la trasformación y el cambio. En la representación funciona como hilo conector del tejido social, significando las prácticas cotidianas.

4. La representación social trata del sentido común. ¿Qué es el sentido común? Ampliamente se acepta la idea de concebirlo como la forma de conocimiento opuesta al saber científico. No se trata entonces de lo empíricamente demostrado, o metodológicamente falseado. Se trata de la experiencia del sujeto corriente, de la interpretación silvestre, espontánea y, si se quiere, ingenua de la vida, sus vicisitudes y finalidades. Ubicar la atmósfera del sentido común es ir al núcleo de la representación. Es otorgarle un carácter cercano y dinámico. Íntimo. En esta perspectiva, el estudio de las representaciones tiene una mirada intuitiva del hacer y ser diario.

5. Las representaciones sociales son comunicación. Es sabido que comunicar implica el intercambio de sentimientos, opiniones o cualquier otro tipo de información mediante el habla, escritura, señas y demás formas. Las representaciones permiten la comunicación, dado su 
Representaciones sociales que tienen los docentes del programa de Educación Física de la Universidad Pedagógica Nacional sobre las competencias propias de su saber disciplinar

Luis Carlos Pérez Ferro, Martha Arenas Molina y Sonia López Domínguez

carácter social. Es a través de ellas como estructuramos el mundo. La comunicación es su vehículo.

6. Las representaciones son desarrollo. En tanto desarrollo, las representaciones modifican la cognición social. Ellas, que a su vez son cognición, figuran el aprendizaje social. La cantidad y calidad de información y experiencia acumulada por la humanidad se actualiza mediante la representación. Aprendemos de nuestras formas de organizar el mundo, de hacerlo comprensible.

Algunas consideraciones metodológicas de la representación social

Como se mencionó antes, en la forma de aplicar la teoría existen aproximaciones diferentes. La variada función de lo anterior provee a las representaciones de una riqueza importante $\mathrm{y}$, al mismo tiempo, hace que cada investigador encuentre su propio camino en el mundo de lo objetivo, en la validez y confiabilidad de los resultados. A continuación se enuncian algunas ideas generales identificadas en trabajos de corte empírico:

1. Énfasis en epistemologías comprensivas: se ha observado que las propuestas metodológicas son cualitativas. Buscan tener un nivel de generalización limitado y no preferencia por la identidad relacional de causas y efectos.

2. Privilegio de instrumentos tradicionalmente cualitativos: son instrumentos aquellos mecanismos y/o dispositivos que permiten capturar y sistematizar la información proveniente de los sujetos sociales. Ellos permiten una exploración silvestre de la arquitectura cognitiva, no son diseñados experimentalmente y hacen de la subjetividad una fortaleza.

3. Necesidad de identificar el contenido, la estructura y núcleo de la representación: los trabajos empíricos coinciden en la identificación de estos tres aspectos presentados como esenciales en la cualificación de la representación social. Es necesario ubicar en los estudios lo que la representación constituye, su sistema característico y su organización central.

\section{Método}

El método utilizado es el plurimetodológico, que en palabras de Abric (1989) significa el uso de varias técnicas, entre ellas métodos interrogativos, que consisten en recoger una expresión de los individuos que afecta al objeto de representación en estudio, y métodos asociativos, que se basan en la expresión verbal.

Tipo de estudio

Descriptivo-explicativo. Busca, en primera instancia, describir y explicar las razones del comportamiento humano, esto referido al caso de cómo piensan los sujetos frente al concepto de competencias. Además, la investigación se hace explicativa, porque proporciona un sentido de entendimiento o de explicación sobre las representaciones.

\section{Proceso}

El estudio se divide en dos etapas: identificar los elementos de la representación de las competencias y conocer la organización de esos elementos e identificar el núcleo central de la representación de las competencias.

\section{Participantes}

Se invitó a un grupo de diez docentes que en la actualidad hacen parte de la planta profesoral del proyecto curricular de la Licenciatura en Educación Física (en adelante PCLEF) de la Universidad Pedagógica Nacional. Los docentes hacen sus prácticas pedagógicas en las diferentes áreas propuestas por el PCLEF: humanística, pedagógica y disciplinar.

\section{Instrumentos}

Los instrumentos diseñados para la recolección de la información fueron: el cuestionario, la carta asociativa y los tris jerárquicos.

El cuestionario: Abric (1989) señala que pertenece al método interrogativo y es la técnica más utilizada en el estudio de las representaciones. El cuestionario aplicado se estructuró en seis categorías: interacción, cognición social, conciencia social, sentido común, comunicación y desarrollo. Cada una de ellas estaba conformada por dos preguntas que permitieron recoger la expresión de los participantes frente al objeto de representación en estudio -las competencias-. Esta técnica permitió identificar la organización y la significación del núcleo central de la representación.

La carta asociativa: método de recolección por asociación donde prima la expresión verbal, de manera espontánea. Los pasos para trabajar el método asociativo, en palabras de Jaoui (1979), citado por Abric en Metodología de recolección de las representaciones sociales, son: en un primer momento y a partir del concepto inductor, se hacen asociaciones libres. Luego 
se pide al participante producir otra serie de asociaciones, teniendo en cuenta una pareja de palabras dentro de las que esté presente el término inductor, además de todas las palabras que por asociación se produjeron en el primer momento.

Tris jerárquicos sucesivos: en palabras de Abric (1989), el método, en la primera parte, consiste en recoger una serie de asociaciones referidas al término inductor -competencias-. Paso seguido, el participante produce una serie de asociaciones referidas al término inductor. Posteriormente, dentro del conjunto de las asociaciones producidas se seleccionan los conceptos más reiterativos que conformaran el corpus de análisis.

En la segunda parte se le muestra al participante la selección de conceptos del corpus; seguidamente, se le solicita que divida el listado de conceptos en dos grupos: un grupo con los conceptos con los que más relaciona el término inductor, y otro con los conceptos con que menos relaciona el término inductor.

Este mismo ejercicio de dividir en dos grupos los conceptos con los que más y los conceptos con los que menos se relaciona el término inductor, se debe repetir hasta que queden, finalmente, los dos conceptos que más se relacionan con el término inductor.

Finalmente, lo que se tiene es una clasificación por orden de importancia de los conceptos relacionados con el término inductor, lo que permite entrar en la fase de análisis de las asociaciones que se han originado.

\section{Resultados}

En primera instancia, la puesta en evidencia del contenido, es decir de los elementos que organizan y proporcionan la significación de la representación, se logró con la aplicación del cuestionario. Se registraron las siguientes respuestas en los participantes:

Tabla 1. Respuestas del cuestionario

\begin{tabular}{l|l|}
\hline $\begin{array}{l}\text { Preguntas cuestionario } \\
\text { ¿Hoy qué se "dice" } \\
\text { debe hacer profesio- } \\
\text { nalmente un educador } \\
\text { físico? }\end{array}$ & $\begin{array}{l}\text { Convocar desde su discurso, que debe ser de tipo } \\
\text { Formentativo, propositivo, proyectivo. }\end{array}$ \\
\hline $\begin{array}{l}\text { Fes, formación crítico-reflexiva, destrezas. } \\
\text { Formar personas con responsabilidad del medio } \\
\text { ambiente. }\end{array}$ \\
\hline $\begin{array}{l}\text { ¿Qué sabe y qué ha } \\
\text { escuchado sobre los } \\
\text { desempeños y tareas } \\
\text { de los profesionales de } \\
\text { la Educación Física? }\end{array}$ & $\begin{array}{l}\text { Se desempeñan en escenarios escolares formales } \\
\text { e informales, en educación especial, técnicos, } \\
\text { rehabilitación, recreadores, entrenadores, escuelas } \\
\text { deportivas, administradores, recreación y deporte. }\end{array}$ \\
\hline
\end{tabular}

¿Desde la perspectiva de la percepción social, es decir, desde el punto de vista de los demás, qué se piensa de nuestra profesión?

¿Cuál es el discurso que usted maneja de la Educación Física?

¿Cuál es el propósito social más importante del educador físico?

Argumente su opinión.

Dentro del amplio espectro de conceptos que se tienen de la Educación Física, ¿con cuál se identifica más? Describa.

En el "colegaje", es decir, en la comunicación que establece con otros educadores físicos ¿qué ha escuchado sobre en que se están desempeñando los egresados?

Si usted pudiera adentrarse en la mente de los administrativos y demás miembros de la universidad no académica, ¿cuál sería el concepto de estos en relación a lo que hacen diariamente los educadores físicos?

¿Cómo presentan los medios masivos de comunicación, en tanto profesión, a la Educación Física?

¿Qué ve y escucha la opinión pública con relación a lo que hacen los educadores físicos?
La Educación Física es una profesión fácil, con poca credibilidad, irrelevante, desacreditada.

Donde las personas se imaginan que allí solo se juega.

Difícil por las condiciones de trabajo.

En algunas ocasiones se reconoce la importancia en el ámbito social.

La Educación Física como profesión está en crisis. La Educación Física contribuya a la adquisición de vida.

La Educación Física refiere a la educación del movimiento, desarrollo motor, capacidades físicas.

La Educación Física como múltiples dinámicas donde se vivencia actividades de juego.

La Educación Física en la relación salud cuerpomente.

La Educación Física requiere modelos alternativos con participación de estudiantes.

Conocer y transformar la sociedad.

Formar para la cooperación desinteresada.

Formar seres humanos.

Función social del valor por el cuerpo.

Cuerpo como construcción social. Educación Física para educar.

EF como práctica social.

EF incluyente que se preocupe por el cuerpo simbólico.

El hombre en movimiento.

EF para mejorar seres humanos y sus habilidades motrices.

EF para el desarrollo integral.

Conjunto de prácticas corporales.

Docencia, escuelas de formación deportiva, gimnasios, discapacitados, entrenadores, administrativos, administración del deporte, juzgamiento deportivo, cajas de compensación, gimnasios, ONG, preparación física, deportes y naturaleza, empresas deportivas y recreativas, investigadores, centros médicos, ciclorrutas, gimnasios, parques.

Los educadores físicos son mirados como un recreador, se cuestiona su saber específico.

Han estudiado EF como una última posibilidad de poder estudiar.

Enseñan deportes y recreación.

Trabajan en el patio y el trabajo es práctico, no teórico.

Se cree que no hacen mucho.

Educan el cuerpo y son activos, juegan.

El programa radial "Por los campos de la EF" presenta profesores protagonistas de innovaciones pedagógicas.

TV y periódicos utilizan la EF como medio para vender aparatos ergonómicos y de fuerza.

El deporte polariza, la TV es social.

Profesionales que interactúan con el área de la salud, poco se ve como disciplina académica. La EF como profesión no es reconocida por los medios, en algunos casos se destacan los entrenadores $\mathrm{y}$ deportistas.

Existe falta de orientación en la clase de EF

La EF es una asignatura fácil.

El educador físico es poco creativo, recursivo, rutinario.

Solo se destacan los resultados deportivos.

Enseñan a jugar los deportes, trabajan la parte básica de la técnica.

Concepto poco favorable, dedicado a la salud del cuerpo.

Se refieren a deportes, actividades en colegios y

escuelas deportivas.

Profesión compleja. 


\begin{tabular}{|c|c|}
\hline $\begin{array}{l}\text { ¿Es posible educar al } \\
\text { cuerpo? Argumente su } \\
\text { opinión. }\end{array}$ & $\begin{array}{l}\text { Se puede educar al cuerpo como despliegue para } \\
\text { la libertad del movimiento y la danza y educar el } \\
\text { cuerpo como repliegue, es decir EF limitante, sin } \\
\text { libertad. } \\
\text { Educar el cuerpo es transformarlo, reflexionar } \\
\text { sobre él. } \\
\text { Educar al hombre es educar al cuerpo. El cuerpo es } \\
\text { un todo humano totalizante, capaz de ser educado. } \\
\text { No educar el cuerpo, pero si los movimientos que se } \\
\text { realizan con el cuerpo. } \\
\text { El cuerpo es educable en hábitos, salud, ejercicios, } \\
\text { alimentación, corporalidad. } \\
\text { El cuerpo se educa en las relaciones con los otros, } \\
\text { es cultural. } \\
\text { El cuerpo no se educa, se generan prácticas corpora- } \\
\text { les que hacen posible la educación del sujeto. }\end{array}$ \\
\hline $\begin{array}{l}\text { ¿Cuál es la utilidad } \\
\text { práctica de la Educa- } \\
\text { ción Física? }\end{array}$ & $\begin{array}{l}\text { Desarrollo humano. } \\
\text { Transformar la realidad y mejorar la convivencia. } \\
\text { Educar para la vida en la actividad física y expresiva, } \\
\text { con sentido social. } \\
\text { Conectar al hombre con la realidad sociocultural. } \\
\text { Socio-afectividad. } \\
\text { Sujetos reflexivos sobre lo que hacen y para qué lo } \\
\text { hacen. }\end{array}$ \\
\hline
\end{tabular}

Tomado del cuestionario

Como se ve, las representaciones sobre el concepto de las competencias propias de los educadores físicos se construyen en la cotidianidad, en sus prácticas pedagógicas y en las relaciones que tejen con sus estudiantes y colegas, es decir, en el diario vivir. Al hacerse comunes, rutinarias y familiares, adquieren sentido para una comunidad, se validan a través de la comunicación. De allí las connotaciones que expresan los entrevistados.

En la etapa denominada, en palabras en Abric (2004), búsqueda del núcleo central, fue necesario referirse a la indagación de la organización de ese contenido en un sistema de categorías, lo que constituye el esqueleto de la representación.

El tratamiento de la información estuvo relacionado con elementos de las categorías gramaticales como nombres, verbos, adjetivos y adverbios. La categoría gramatical más frecuente para asociar el corpus de competencia son los nombres.

Por otro lado, Abric (2004) considera que la identificación de los lazos de las relaciones y la jerarquía entre los elementos, permite entrar a la organización interna de la representación. Así, el ejercicio llevado a cabo permite colegir lo siguiente: los cinco docentes reiteraron en el primer ejercicio de la agrupación de los dieciséis términos más relacionados con el corpus de competencias, el uso de nombres como: autocomunicativa, proyecto de vida, pensamiento complejo, pluridiversa, multidiversa, interpersonal, intrapersonal, conocimiento, inteligencia, entendimiento, maestría, formación, valores, motriz, operación mental, saber, técnica, académica, personales, conceptuales, relaciones-medio, cultura, sociedad, procedimentales, social. Los nombres en mención están relacionados con la experiencia y el rol de los docentes, pero también con las competencias actuales planteadas por el PCLEF.

A continuación se presenta un paralelo entre las competencias actuales del PCLEF y el primer ejercicio de la agrupación de los dieciséis términos con que más asocian al corpus de competencia:

Tabla 2. Paralelo competencias PCLEF - Categoría nombres

\begin{tabular}{|c|c|}
\hline Competencias básicas PCLEF & $\begin{array}{l}\text { Categoría nombres asociados al } \\
\text { corpus de competencia }\end{array}$ \\
\hline $\begin{array}{l}\text { - Cognitivas: interpretativa, argumenta- } \\
\text { tiva, comprensiva, propositiva. } \\
\text { · } \quad \text { Comunicativas. } \\
\text { - } \quad \text { Autogestión del proyecto ético de vida. } \\
\text { - } \quad \text { Orientación de servicio. } \\
\text { - } \quad \text { Pensamiento complejo. } \\
\text { - } \text { Autonomía. } \\
\text { - Metacognición. }\end{array}$ & $\begin{array}{l}\text { Autocomunicativa. } \\
\text { Proyecto de vida. } \\
\text { Pensamiento complejo. } \\
\text { Conocimiento. } \\
\text { Inteligencia. } \\
\text { Entendimiento. } \\
\text { Operación mental. } \\
\text { Relaciones medio ambiente. } \\
\text { Interpersonal, intrapersonal. } \\
\text { Formación. } \\
\text { Valores. }\end{array}$ \\
\hline \multicolumn{2}{|l|}{ Competencias genéricas PCLEF } \\
\hline 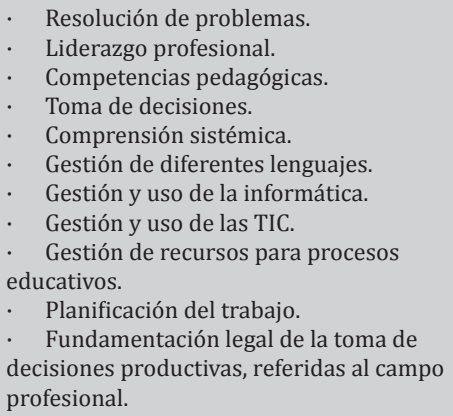 & $\begin{array}{l}\text { Los nombres utilizados por los } \\
\text { docentes para asociar términos } \\
\text { al corpus de competencia no se } \\
\text { identifican. }\end{array}$ \\
\hline \multicolumn{2}{|l|}{ Competencias específicas PCLEF } \\
\hline $\begin{array}{l}\text { Fundamentación científica, pedagógica, } \\
\text { disciplinar, reflexiva de las prácticas desde } \\
\text { el objeto de estudio de la licenciatura. } \\
\text { Gestión del proyecto de vida en relación } \\
\text { con la EF. } \\
\text { Comunicativa específica. } \\
\text { Evaluar los procesos educativos concer- } \\
\text { nientes a la EF. } \\
\text { Proyectar, planificar y ejecutar procesos } \\
\text { educativos. } \\
\text { Formular proyectos de investigación. }\end{array}$ & $\begin{array}{l}\text { Pluridiversidad. } \\
\text { Multidiversidad. } \\
\text { Motriz. } \\
\text { Saber. } \\
\text { Técnica. }\end{array}$ \\
\hline
\end{tabular}

Tomado de competencias PCLEF y tris jerárquicos

Así, para poder evidenciar el resultado final del ejercicio de los tris respecto a la asociación con el corpus de competencia, se mostrará en el siguiente paralelo el resultado del ejercicio donde los docentes seleccionaron un (1) solo término con el que más asocian o el más cercano al corpus de competencia. 
Tabla 3. Competencias PCLEF - Corpus competencia

\begin{tabular}{|l|c|}
\hline Competencias PCLEF & $\begin{array}{c}\text { Último término con el cual asocian el corpus de } \\
\text { competencia }\end{array}$ \\
\hline Competencia Básica & Desarrollo. \\
\hline Competencia Básica & Pensamiento complejo. \\
\hline Competencia Básica & Capacidad. \\
\hline Competencia Básica & Proyecto de vida. \\
\hline
\end{tabular}

Tomado de competencias PCLEF y tris jerárquicos

\section{Discusión}

Las siguientes consideraciones ponen de relieve la comparación y postura crítica entre los núcleos "nombres-palabras" que constituyen el cuerpo de la representación, y las competencias que el proyecto curricular Licenciatura en Educación Física ha formulado como ideario de formación. Es, entonces, un partir de los hallazgos "nombres-palabras" para llegar a lo establecido y, desde allí, formular comentarios en tono de preguntas, cuya posibilidad de resolución pueda ser insumo de desarrollo estratégico en el programa y la Facultad.

Lo representado en torno a las competencias propias que tienen los licenciados de Educación Física sobre su saber disciplinar, agrupa conceptos como: desarrollo, pensamiento complejo, capacidad y proyecto de vida. Son, pues, un corpus representacional que se ha configurado como una forma de cognición y/o pensamiento social que tiene su asidero en los corredores y campus de la Facultad. El lenguaje común utilizado entre maestros se figura como afluente de la representación. Ya lo mencionaría la teoría de las representaciones: lo expresado en la cotidianidad es un modo de comunicación que prepara y anticipa la representación.

La noción de representación social tiene que ver con la manera como nosotros, sujetos sociales, aprehendemos los acontecimientos de la vida diaria, las características de nuestro medio ambiente, las informaciones que en él circulan y la significación de las personas de nuestro entorno próximo y lejano (Pérez, 2005).

Desde el punto de vista del desarrollo es necesario mencionar que este aspecto implica tener en consideración múltiples miradas, que discurren desde los enfoques innatistas hasta los de orden social y culturalista. La mayoría comparten la idea de construcción progresiva de saberes y destrezas, a partir de estadios de menor complejidad a mayor complejidad. Ese principio, tan en boga a partir de los años cincuenta, proporciona una lectura diferente sobre las competen- cias existentes en el programa de Educación Física. La pregunta sería: ¿cuál es el modelo de desarrollo que sostiene la enunciación de esas competencias? ¿Es un enfoque invariable del desarrollo? ¿Evolucionista?

Si se acepta que es posible enunciar competencias dentro de un proceso de formación, distintas en cuanto categoría y jerarquía unas de otras -básicas, genéricas y específicas, planteadas por el programa-, entonces, ¿Cómo se observarían los cambios? ¿Qué mecanismo permitiría eso? ¿Es posible medir una misma competencia a través de la modificación de sus desempeños, variando su naturaleza?

De otro lado, y desde una concepción desarrollista del aprendizaje, sería posible argumentar que existen relaciones entre las distintas competencias -básicas, genéricas, específicas- en términos de condición, es decir, $¿$ se sucede una a la otra en orden progresivo? ¿O aparecen al tiempo?

Sobre el pensamiento complejo, que se observa como categoría principal del contenido de la representación en este trabajo, señalamos que se incluye como competencia básica en el programa de Educación Física de la UPN. Sin embargo, no existe en las competencias que acompañan a las básicas, es decir, las genéricas y las específicas. En otras palabras, no hay descriptores, aspectos y/o desempeños que continúen su desarrollo. Se plantean competencias nuevas que fracturan su continuidad.

Encontramos la noción de complejidad y pensamiento complejo en el filósofo francés Edgar Morin (1979), quien en palabras sencillas se refiere a él como la capacidad de interconectar distintas dimensiones de lo real, ante la emergencia de hechos u objetos multidimensionales. Pero, ¿cómo es posible medir u observar el pensamiento complejo en tanto competencia? ¿Cuáles serían sus desempeños particulares? Y sobre todo, ¿Cómo se orquestaría esa competencia con el objeto de análisis de la Educación Física, expresado también en cuanto competencia?

La capacidad ocupa otro importante escaño en las representaciones que tienen los maestros sobre la competencia profesional. Tener capacidad es ser competente profesionalmente. Pero, ¿cómo se logra una buena capacidad profesional? 0 mejor, ¿cuándo se es capaz? ¿Qué se necesita para ello? En relación, se podría decir que el programa de Educación Física de la UPN considera que la apuesta hecha por las compe- 
Representaciones sociales que tienen los docentes del programa de Educación Física de la Universidad

Pedagógica Nacional sobre las competencias propias de su saber disciplinar

Luis Carlos Pérez Ferro, Martha Arenas Molina y Sonia López Domínguez

tencias que ha formulado crean las condiciones para el trabajo. Pero este escenario actualiza la competencia, la reestructura. ¿Cómo lo hace? Y, ¿cómo se retroalimentan recíprocamente?

Finalmente, el proyecto de vida constituye el último elemento del sistema de conceptos propios al contenido de la representación. Las competencias profesionales son proyecto de vida. Ya se observa en el programa, dentro de las denominadas competencias básicas, una noción semejante. Se trata de la autogestión del proyecto ético de vida. Lo que ocurre es que ella no aparece desarrollada consecuentemente en la competencias más "complejas" -genéricas y específicas-.

\section{Conclusiones}

Los participantes -profesores del programa Licenciatura en Educación Física de la UPN- asocian el concepto de competencia profesional con la categoría gramatical de nombres, siendo los más cercanos al término inductor: desarrollo, pensamiento complejo, conocimiento, capacidad y proyecto de vida. En este sentido, la propuesta del teórico Moscovici cobra significado cuando se refiere a las representaciones sociales como parte del mundo de la interacción, es decir, que el concepto de competencia es una propuesta de pensamiento social donde se comparten modos de pensar y actuar. Allí el otro es indispensable, pues es con él y mediante ello como se construyen, trasmutan y establecen los conceptos de competencias del licenciado de Educación Física, todo esto a través del entramado social propio del programa.

Concibiéndose en palabras de Moscovici, las representaciones son una forma de conocimiento cotidiano, es decir, de la experiencia del sujeto corriente, de la interpretación silvestre, del sentido común, espontáneo y, si se quiere, una mirada intuitiva del hacer y ser diario. Entonces, desde esta perspectiva, la representación social del corpus de competencia respecto al hacer de los docentes y desde la óptica social, cobra sentido cuando los docentes son representados en los imaginarios sociales como las personas que se dedican a jugar, trabajar con el cuerpo y el movimiento, mejorar las relaciones sociales y a enseñar deportes y recreación.

El proyecto curricular de la Licenciatura en Educación Física (PCLEF), en su propósito por la formación integral de los estudiantes y de su contribución en la transformación social, permea la mente de los docentes cuando estos asocian el término inductor compe- tencias con conceptos como: social, medio ambiente, dimensiones, convivencia, autocontrol, trabajo en equipo. Aquí toma fuerza, en palabras de Moscovici -las representaciones sociales son comunicación-, ya que es sabido que comunicar implica el intercambio de sentimientos, opiniones o cualquier otro tipo de información mediante el habla, escritura, señas y demás formas. Las representaciones permiten la comunicación, dado su carácter social. Es a través de ellas como estructuramos el mundo y adquiere sentido.

Los nombres con que los docentes relacionan el término inductor -competencias- están relacionados con la experiencia y el rol de los docentes, pero también con las competencias actuales planteadas por el proyecto curricular de la Licenciatura en Educación Física, al referirse a competencias cognitivas, comunicativas, de autogestión, orientación del servicio y al pensamiento complejo.

Desde el punto de vista del conocimiento, los docentes inscriben las competencias en el denominado sabersaber. Sin embargo, complementan este aspecto con el saber-ser y saber-hacer. Es decir, todas comparten el saber como eje común. Esto denota la profunda insistencia por desarrollar las competencias básicas-cognitivas propuestas en el proyecto curricular de la Licenciatura en Educación Física.

La elección de la profesión y sus roles son una opción vital; esto en tanto caracterizará el perfil del sujeto durante toda su existencia. Es quizás por esa razón que los profesores participantes, y luego del tratamiento de la información, consideran que la competencia profesional es un proyecto de vida. Esto en consecuencia al carácter desarrollista de la representación. Son, pues, desarrollo. Son proyecto vital.

\section{Referencias}

Abric, J. (1989). Metodología de recolección de las representaciones sociales. En Practiques sociales et représentations. Traducción al español por José Dacosta y Fátima Flores (2001). Prácticas sociales y representaciones sociales. México: Ediciones Coyoacán.

Abric, J. (2004). Prácticas sociales y representaciones. México D.F.: Ediciones Coyoacán.

Jaoui, H. (1979). Técnica de la carta mental. México, D.F.: Ediciones Coyoacán.

Jaramillo, J. et al. (2009). Proyecto Curricular Licenciatura en Educación Física, PCLEF. Bogotá: Universidad Pedagógica Nacional. Facultad de Educación Física. 
Mora, M. (2002). La teoría de las representaciones sociales de Serge Moscovici. Recuperado el 14 de septiembre de 2012 en athenea/num2/Mora.pdf

Morín, E. (1990). Introducción al pensamiento complejo. Bogotá: Editorial Gedisa.

Moscovici, S. (1979). El psicoanálisis, su imagen y su público. Buenos Aires: Editorial Huemul S.A.

Pérez, L. (2005). Representaciones sociales del desarrollo y la gestión de la investigación. El caso de la Facultad de Psicología de la Universidad Cooperativa de Colombia. Bogotá: Pontificia Universidad Javeriana. Desarrollo y Gestión Investigativa. 